\title{
The Gouy phase shift in terahertz time-domain spectroscopy and its experimental estimation, modelling and compensation
}

\author{
Pierre Koleják ${ }^{1,2}$, Kamil Postava ${ }^{1}$, Martin Mičica ${ }^{1,2}$, Mathias Vanwolleghem ${ }^{2}$ and Jaromír Pištora ${ }^{1}$ \\ ${ }^{1}$ VSB-Technical University of Ostrava, Nanotechnology centre and IT4Innovations, \\ 17. listopadu 15, 70833 Ostrava-Poruba, Czech Republic \\ ${ }^{2}$ Université de Lille, CNRS UMR 8520, Institut d'Electronique, de Microelectronique et de Nanotechnologie, \\ 59652 Villeneuve d'Ascq Cedex, France
}

\begin{abstract}
Spatial properties of pulsed beams become more important in the terahertz range, which causes that reference and sample measurements can differ in the spectral dependent complex phase called the Gouy phase. It originates from propagation of the beam wavefront close to the focus. In such a case, the plane wave approximation has not to be fulfilled and measured data gives incorrect absorptions and dispersions. We present techniques to compensate these phenomena as well as the procedure to achieve the Gouy phase from measured data. Moreover, the model to simulate the Gouy phase detection will be shown in this paper. It gives powerful tool to avoid experimental errors caused by the Gouy phase in terahertz time-domain spectroscopy.
\end{abstract}

\section{INTRODUCTION}

$\mathrm{T}$ he terahertz time-domain spectroscopy (THz-TDS) is phase sensitive technique able to determine complete complex dielectric function of a measured sample from a single transmission spectrum. THz-TDS spectrometers use sub 100 -femtosecond pulsed beams. If one takes into account relatively long wavelength of the radiation, the terahertz beams are more tightly focused in contrast with longer wavelengths as the visible beams.

It is necessary to carry out reference and sample measurement in THz-TDS measurement. If optical paths of $\mathrm{THz}$ beams are the same for reference and sample measurement, the beams are focused in the same way on a detector for both measurements. It means also the same Gouy phases [1].

However, if the optical paths differ, especially for sample measurement including a propagation through a sample, the focusing of the $\mathrm{THz}$ beam on detector is changed for the sample measurement in contrast with reference measurement. Therefore, reference and sample measurements will have different complex Gouy phases, which affects measured transmission spectra.

\section{COMPENSATION OF THE Gouy PHASE}

The Gouy phase influence have to be compensated for obtaining the correct dielectric function of a sample. The numerical method to compensate the Gouy phase was proposed by Kužel et al. [2], which uses linear approximation of the Gouy phase, where only the real part of the Gouy phase influences measurement. In a case, that the Gouy phase has a general dependence, it is more advantageous to use another technique. In our previous paper [3], we have proposed the mechanical method for compensation of the complex Gouy phase. The method is applicable for strongly focused $\mathrm{THz}$ beams of general shape and included misalignments of the experimental setup.
Figure 1 shows comparison of uncompensated and compensated complex refractive index of high resistivity silicon measured in transmission setup. It is visible that uncompensated data include unphysical absorptions and dispersions.

The time domain spectrometer Teraview TPS Spectra 3000 is used for measurement in this paper. It uses $90 \mathrm{fs}$ pulses at 780 $\mathrm{nm}$ generated by Er:doped fiber laser. The THz emission as well as detection are delivered by GaAs photoswitches. Measurements were carried out in the vacuum.

In this contribution, we present also technique for analyzing of errors for general data caused by the complex Gouy phase. It allows one to determine it is necessary to use the compensation methods. Also, the comparison of measured complex Gouy phase with the numerical model to simulate its detection on a finite size detector [4] is provided. It allows one to minimize the Gouy phase influence in spectroscopic measurements and helps to design terahertz time-domain spectrometer setups with reduced the Gouy phase shift.
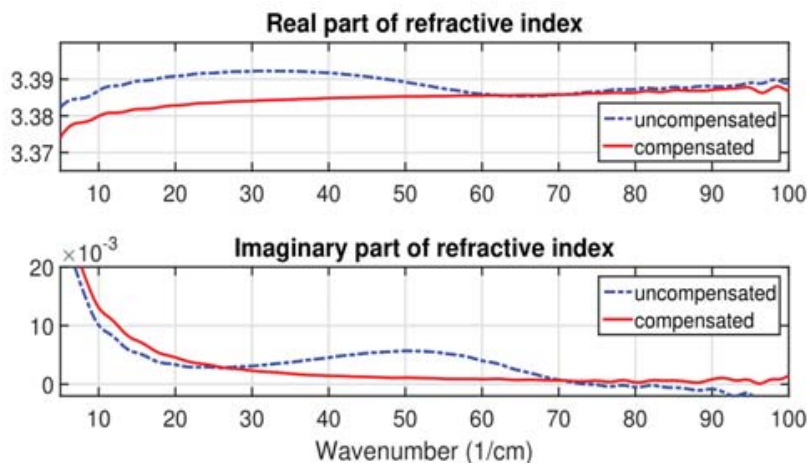

Fig. 1 Real and imaginary part of complex refractive index of high resistivity silicon are shown. Uncompensated (dashed blue line) and compensated (full red line) complex refractive indices differ in the Gouy phase influence [3].

\section{MODEL OF THE GOUY PHASE}

The complex Gouy phase $[1,3]$ is the additional axial complex phase of the Gaussian beam caused by its diffraction, expressed for propagating along z-axis in form

$$
\tilde{\zeta}_{c}(z)=\arctan \left(\frac{z}{z_{0}}\right)+\mathrm{j} \ln \sqrt{z^{2}+z_{0}^{2}},
$$

where $z_{0}$ denotes the Rayleigh parameter. The real part represents the different phase velocity between Gaussian beam and plane wavefronts and affects the extraction of the real part 
of sample refractive index. Whereas, the imaginary part is associated with amplitude changes caused by the beam focusing.

The complex Gouy phase, affecting the sample measurement as is shown on Fig. 1, may be obtained from the measured data as the complex spectra ratio of the free space measurement on the standard reference z-position and the free space measured on the z-position corresponding to the focus displacement caused by a sample [3]. It is important to note, that the standard reference position may be placed out of the beam focus. Which means the extracted complex Gouy phase by this way corresponds to the difference of complex Gouy phases on different z-position of the beam.

Moreover, the detection is carried out by a finite size detecting area. Which will also cause the detection of nonaxial components of the Gaussian beam averaged on a detection area [4]. The model for describing of the averaged complex Gouy phase $\left\langle\widetilde{\zeta_{c}}(\Omega, z)\right\rangle$ uses the integral averaging of the complex Gaussian amplitude on a finite circular area perpendicular to the beam axis which passes thought the detector center [4] and gives the form

$$
\left\langle\widetilde{\zeta_{c}}(\Omega, z)\right\rangle=-\mathrm{j} \ln \left\{\frac{\exp \left(\frac{-\mathrm{j} k R^{2}}{2\left[z^{\prime}+\mathrm{j} z_{0}(\Omega)\right]}\right)-1}{\exp \left(\frac{-\mathrm{j} k R^{2}}{2\left[z_{0}^{\prime}+\mathrm{j} z_{0}(\Omega)\right]}\right)-1}\right\}
$$

where $\Omega, k, R, z_{0}^{\prime}, z^{\prime}$ mean the frequency, the wavenumber in the vacuum, the radius of a detector, the reference z-position, and the displaced z-position, respectively.

The complex Gouy phase extracted for the high resistivity silicon measurement previously shown in Fig. 1 is plotted (blue curve) in Fig. 2. The measured complex Gouy phase allows one to see whether the dependence of the real part of the Gouy phase is linear. In this case, it is possible to use the compensation method proposed by Kužel at al. [2] based on the numerical compensation considering the linear dependence of the real part with zero value of the imaginary part of the Gouy phase.
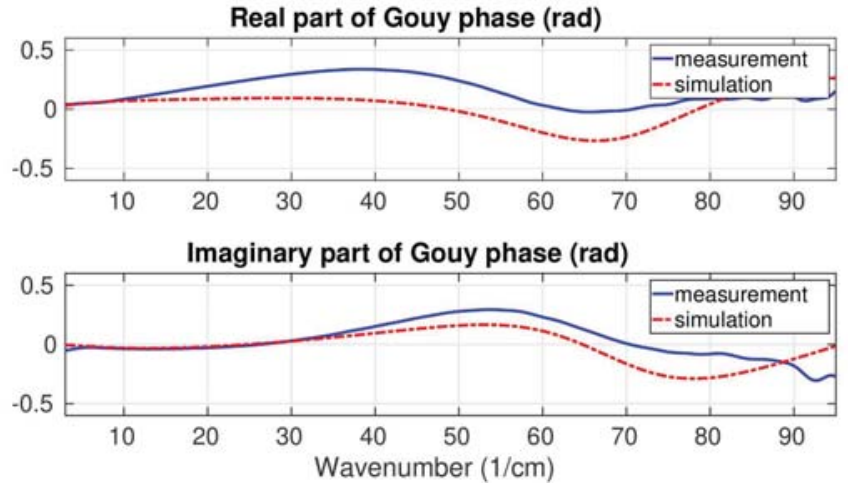

Fig. 2 Comparison of the measurement (blue curve) and simulation (red dashed curve) of the complex Gouy phase corresponding to the measurement of the high resistivity silicon.

Figure 2 shows that the dependence is strongly nonlinear and the imaginary part of the Gouy phase also strongly affects measurement. It follows it is advantageous to use the mechanical method [3] for measurements on this experimental setup. Generally, the nonlinear behavior may be caused also by the misalignment of the system or another effect such as non-Gaussian beam etc. Moreover, these effects are difficult to model, but the mechanical method is principally able to compensate most of them due to achieving information about how the reference is changing along z-axis. Disadvantages of the mechanical method in contrast with the numerical method is that data are compensated for the arbitrary position corresponding to the refractive index on the arbitrary position, which limits application of the method for materials with huge dispersion in broad terahertz spectral range.

The model simulating the averaged complex Gouy phase $\left\langle\widetilde{\zeta_{c}}(\Omega, z)\right\rangle$ is shown in Fig. 2 (dashed red curve). Input parameters used in the simulation are $R=1.5 \mathrm{~mm}, z^{\prime}=7.95 \mathrm{~mm}$, $z_{0}^{\prime}=6.6 \mathrm{~mm}$ and $z_{0}=\frac{6.76}{\Omega}(\mathrm{mm}, \mathrm{Hz})$. The model describes the measured Gouy phase roughly due to approximations in its deriving [4], but still able to demonstrate that observed phenomena may be caused by the behavior of the Gaussian beam in $\mathrm{THz}$ frequencies.

\section{CONCLUSION}

We propose techniques for compensation of the Gouy phase, which affects transmission time-domain spectroscopy measurements. The method to analyze general influence of the Gouy phase is proposed. The model simulating the Gouy phase influence compared with measured data is also shown. These analyses lead to understand and compensate errors originated from the Gouy phase in THz-TDS systems. More detailed study of these errors will be published in our following work [5].

\section{ACKNOWLEDGEMENT}

Partial support from the projects 18-22102S from Czech Science Foundation, SP2019/92 project, CZ 02.1.01/0.0/0.0/16 013/0001791, the Czech-French mobility project 8J19FR006, and Scholarship of the city of Ostrava are acknowledged.

\section{REFERENCES}

[1]. S. M. Feng and H. G. Winful, "Physical origin of the Gouy phase shift," Optics Letters, vol. 26, pp. 485-487, APR, 2001.

[2]. P. Kužel et al., "Gouy shift correction for highly accurate refractive index retrieval in time-domain terahertz spectroscopy," Opt. Express, vol. 18, pp. 15338-15348, JUL, 2010.

[3]. P. Koleják et al., "Experimental Gouy phase shift compensation in Terahertz time-domain spectroscopy," Phot. Nanostr, vol. 31, pp. 129-133, 2018.

[4]. P. Koleják et al., "Analytical model of Gouy phase influence in terahertz time-domain spectroscopy,” Proceedings of SPIE, vol. 10976, 2018.

[5]. P. Koleják et al., "Quantification of errors originating from Gouy phase shift in terahertz spectroscopy," (to be submitted). 